\title{
ESTABLECIMIENTO DE CULTIVOS DE COBERTURA Y EXTRACCIÓN TOTAL DE NUTRIENTES EN UN SUELO DE TRÓPICO HÚMEDO EN LA AMAZONÍA PERUANA
}

\section{ESTABLISHMENT OF COVER CROPS AND THEIR GROWTH AND NUTRIENT UPTAKE IN A HUMID TROPICAL SOIL OF THE PERUVIAN AMAZON}

\author{
Fernando Puertas ${ }^{1}$, Enrique Arévalo ${ }^{2,}$ Luis Zúñiga ${ }^{2}$, Julio Alegre ${ }^{3}$, Oscar Loli ${ }^{3}$, Hugo Soplin $^{3}$ y Virupax Baligar ${ }^{4}$
}

Resumen

Con la finalidad de evaluar el establecimiento de cinco cultivos de cobertura y determinar su aporte potencial a la fertilidad del suelo en función a la extracción total de nutrientes, se instaló un experimento en la Estación Experimental "El Choclino" del Instituto de Cultivos Tropicales, ubicado en la región de San Martín, Perú. Cinco cultivos de cobertura fueron sembrados: Arachis pintoi Krapov. \& W.C. Greg, Calopogonium mucunoides (L.), Callisia repens (Jacq.) L., Canavalia ensiformis (L.) y Centrosema macrocarpum Benth. El porcentaje de cobertura alcanzado por Canavalia, fue significativamente superior a los demás cultivos, logrando el cubrimiento total del suelo a los 90 días después de la siembra. La mayor producción de biomasa foliar y radicular sobre la base de materia seca, se consiguió con Centrosema (9.61 t ha-1 y $2.76 \mathrm{t}$ ha-1, respectivamente). Las cantidades de Nitrógeno (311 kg ha-1), Fósforo (24.97 kg ha-1) y Potasio (155.61 kg ha-1) extraídas por Centrosema fue significativamente mayor a las extraídas por las otras coberturas. Para las condiciones del ensayo, Canavalia fue el cultivo de cobertura más apropiado como herramienta de manejo para reducir el crecimiento de vegetación espontanea y proteger al suelo de la erosión al cubrir el suelo en menor tiempo. Para mejorar la fertilidad del suelo a través del reciclaje de nutrientes se recomienda usar Centrosema, por presentar la mayor capacidad extractiva de nutrientes del suelo y producir más cantidad de materia seca.

Palabras claves: porcentaje de cobertura, biomasa total, materia seca, nutrientes

\begin{abstract}
In order to evaluate the establishment of five cover crops and their potential to increase soil fertility through nutrient uptake, an experiment was installed at the Research Station of Choclino, San Martin, Peru. Five cover crops were planted: Arachis pintoi Krapov. \& W.C. Greg, Calopogonium mucunoides (L.), Callisia repens (Jacq.) L., Canavalia ensiformis (L.) and Centrosema macrocarpum Benth. The percentage of cover of Canavalia, was significantly higher than the others, reaching total cover at 90 days after planting. The highest above and below ground dry biomass was for Centrosema (9.61 t ha-1 y $2.76 \mathrm{t}$ ha-1, respectively). The rates of Nitrogen (311 kg ha-1), Phosphorous (24.97 kg ha-1) and Potassium (155.61 kg ha-1) extracted by Centrosema were significatively higher than the other cover crops. Therefore, the most appropriate cover crop under the study conditions was Canavalia allowing it to be used as a management tool to reduce spontaneous vegetation growth and to protect the soil from erosion by covering it in less time. To improve soil fertility through nutrient cycling the use of Centrosema is recommended due to its highest nutrient extraction capacity and dry biomass production.
\end{abstract}

Key words: percentage of cover, total biomass, dry matter, nutrients

\section{Introducción}

La tala ilegal, la agricultura migratoria de tumba y quema para sembrar cultivos y pastos, factores de desarrollo socio económicos, como las nuevas carreteras de penetración y las migraciones en búsqueda de mejores oportunidades de vida, constituyen algunas de las fuerzas o procesos que conducen a la deforestación de la Amazonía. Todas estas actividades son practicadas por colonos, pobladores migrantes y nativos y como consecuencia de ellas se están destruyendo los bosques ya que estas áreas son abandonadas para seguir tumbando y quemando mas foresta (Iturregui, 2007; Palm et al., 2005). Por otro lado el manejo inadecuado de los suelos tropicales sin prácticas conservacionistas y el uso de cultivos ilegales están degradando los suelos de la región oriental del Perú con la pérdida consecuente de sus propiedades físicas, químicas y biológicas. Además no se están usando los suelos de acuerdo a sus potencialidades (García \& Durán, 2000). 
La agroecología propone estrategias capaces de superar las limitaciones estructurales y funcionales inherentes a los sistemas de producción simplificados. Tales estrategias se dirigen hacia la estimulación y optimización de los procesos biológicos del suelo, favoreciendo el reciclaje de nutrientes. En ellas se prioriza la adopción de técnicas multifuncionales que puedan mantener o mejorar la fertilidad del suelo, contrarrestar procesos de erosión, favorecer la presencia de poblaciones de organismos benéficos y controlar el surgimiento de vegetación espontánea.

Entre estas estrategias está el empleo de cultivos de cobertura que son especies con características deseables, en rotación o asociación con cultivos de interés económico (Espindola et al., 2005). Si estas especies son leguminosas - son las que tienen la tasa promedio de crecimiento más rápida $\mathrm{y}$ generalmente contienen una mayor cantidad de nutrientes en comparación con otras especies - se promueve el aporte de nitrógeno al suelo gracias a la simbiosis establecida entre ellas y las bacterias fijadoras de nitrógeno atmosférico. Esto reduce e incluso en algunos casos puede eliminar la necesidad de fertilizantes nitrogenados (Marinho et al., 2007).

En trabajos realizados en la amazonía peruana (Alegre et al., 2003; Reyes \& Ara, 1999; Reyes et al., 2004), se señalan los beneficios de los cultivos de cobertura que han sido manejados en diversos sistemas agrícolas y forestales, que involucran el pijuayo (Bactris gasipaes), palma aceitera (Elaeis guineensis), plátano (Mussa sp.), inga (Inga edulis), colubrina (Colubrina glandulosa), etc., así como también rotaciones con maíz (Zea maíz) y caupí (Vigna unguiculata).

El valor del cultivo de cobertura para mantener la fertilidad del suelo en los diversos sistemas agrícolas depende de la producción razonable de materia seca y la concentración de los nutrientes (Altieri, 1999). Una buena planta para cultivo de cobertura mantiene o mejora las condiciones del suelo al mismo tiempo que satisface las necesidades de manejo y requerimientos del suelo de un sistema agrícola en particular. Para asegurar el éxito del cultivo de cobertura en cualquier sistema agrícola, como primera opción se debe considerar las especies leguminosas bien adaptadas a las condiciones climáticas y de manejo (Baligar et al., 2007).

Los objetivos del presente ensayo fueron: evaluar las características de crecimiento de cinco cultivos de cobertura y determinar su aporte potencial a la fertilidad del suelo.

\section{Materiales y métodos}

Descripción del lugar de ejecución

El ensayo se ejecutó en la Estación Experimental "El Choclino", ubicado a $06^{\circ} 28$ '37.3" de Latitud sur y $76^{\circ} 19^{\prime} 54.6^{\prime}$ 'de Longitud oeste, a una altitud de 506 msnm, distrito de la Banda de Shilcayo, provincia y región de San Martín, Perú. Las actividades del ensayo se iniciaron en Abril del 2006 y finalizaron en Mayo del 2007.

El clima es húmedo tropical, con temperatura promedio anual (Mayo, 2006 - Abril, 2007) de $25.1^{\circ} \mathrm{C}$, con precipitación anual de $1451.7 \mathrm{~mm}$., en el mismo periodo, siendo los meses de Mayo a Septiembre los de menor precipitación.

El experimento se desarrolló sobre un suelo arcilloso, ligeramente ácido, nivel medio en materia orgánica, bajo en fósforo disponible, alto en potasio disponible, alta capacidad de retención de cationes y 99\% de saturación de bases, el mismo que estaba invadido en su mayor parte con gramíneas.

Establecimiento de los tratamientos

Para la siembra de las coberturas, las gramíneas y demás especies que cubrían el suelo, fueron cortadas y eliminadas del campo en forma manual. Después se realizó el muestreo inicial del suelo a una profundidad de $0-20 \mathrm{~cm}$, con un muestreador tipo barreno de 2.5 $\mathrm{cm}$ de diámetro interior. Demarcada el área experimental, se procedió a sembrar como cultivos de cobertura Calopogonium mucunoides, Canavalia ensiformis y Centrosema macrocarpum con semilla botánica y Arachis pintoi y Callisia repens con semilla vegetativa (esquejes), a un distanciamiento de $0.50 \mathrm{~m}$ x $0.50 \mathrm{~m}$, siendo la densidad de siembra igual en los cinco cultivos.

Se utilizó el Diseño de Bloques Completos al Azar (DBCA) con cinco tratamientos y tres repeticiones, realizándose la prueba de Duncan al 5\% de probabilidad para comparar los promedios.

Evaluaciones

a) Porcentaje de cobertura

La evaluación del porcentaje de cobertura fue mediante tomas fotográficas digitales (cuatro por cada parcela), las cuales fueron contrastadas y procesadas mediante el software ASSES. La primera evaluación se realizó a los 30 días después de la siembra, las evaluaciones posteriores se realizaron mensualmente hasta que se alcanzó el $100 \%$ de cobertura.

\section{b) Biomasa foliar}

La determinación de biomasa foliar (hojas y tallos) se efectuó al año de instalado el experimento, excepto en Canavalia ensiformis donde se hizo a los ocho meses por ser una leguminosa de corto periodo vegetativo. Para la evaluación de la biomasa, se escogieron dos puntos al azar dentro de cada parcela (15 parcelas) y se colocó un cuadrante de madera de 1 $\mathrm{m} 2$ recolectándose todo el material vegetal para pesarlo en fresco y luego seleccionar dos sub-muestras de cada parcela para ser llevadas a estufa a una temperatura de $72^{\circ} \mathrm{C}$ por 72 horas. Después de determinar el peso seco de la biomasa aérea, ambas sub-muestras de cada parcela se juntaron para ser molidas, envasadas, etiquetadas y enviadas al laboratorio para su análisis foliar.

c) Biomasa radicular 
Para determinar la biomasa radicular se utilizó dos cuadrantes de madera de $0.25 \mathrm{~m}$ x $0.25 \mathrm{~m}$ cada uno, evaluándose dos puntos al azar dentro del cuadrante usado para evaluar la biomasa foliar. Se recolectaron las raíces de cada cobertura hasta una profundidad de $50 \mathrm{~cm}$, y luego de lavarlas y orearlas a temperatura ambiente, se siguió el mismo procedimiento descrito para biomasa foliar.

d) Profundidad de raíces

La evaluación de la profundidad de las raíces se realizó en cada una de las calicatas de $0.50 \mathrm{~m}$ x $0.80 \mathrm{~m}$ x $0.50 \mathrm{~m}$ de profundidad hechas en cada parcela.

\section{Resultados y discusión}

a) Porcentaje de cobertura del suelo

A los 30 días el porcentaje de cobertura de Canavalia ensiformis fue significativamente superior al de los otros cultivos. A los 60 días hubo un incremento en el porcentaje de cobertura de los cultivos, alcanzando valores entre 14 y $94 \%$ existiendo diferencias estadísticas entre ellos. A los 90 días después de la siembra Canavalia ensiformis alcanzó el $100 \%$ de cobertura, mientras que los demás tratamientos alcanzaron entre 22 a $85 \%$ de cobertura. A los 120 días después de la siembra Calopogonium mucunoides alcanzó el 100\% de cobertura, superando estadísticamente a Centrosema macrocarpum, Callisia repens y Arachis pintoi que alcanzaron 83, 53 y 29\% de cobertura, respectivamente. A los 150 y 180 días $C$. macrocarpum y C. repens, lograron el $100 \%$ de cobertura en comparación con Arachis pintoi que a los 210 días de sembrado logra un 98\% de cobertura, siendo el cultivo más tardío en lograr el completo cubrimiento (Tabla 1). Una de las consideraciones importantes que se debe tener en cuenta para elegir una cobertura es el tiempo o la velocidad con la que cubre y protege la superficie del suelo, bajo esta premisa, Canavalia ensiformis, fue la cobertura que cubrió el suelo en menor tiempo logrando así reducir el crecimiento de vegetación espontanea, proteger al suelo de la erosión y reducir la lixiviación de nutrientes en el suelo. estadísticamente superior a las producidas por los otros tratamientos (Tabla 2), siendo las coberturas de Canavalia ensiformis y Callisia repens las que produjeron menor materia seca $\left(4.59\right.$ y $4.45 \mathrm{t} \mathrm{ha}^{-1}$, respectivamente). Como menciona Altieri (1999), el valor del cultivo de cobertura para mantener la fertilidad del suelo en los diversos sistemas agrícolas depende de la producción razonable de materia seca y su concentración de nutrientes. C. macrocarpum fue la cobertura que logró producir mayor cantidad de materia seca que las otras coberturas.

Tabla 2. Biomasa foliar (materia seca) producida por cinco especies vegetales usadas como cobertura.

\begin{tabular}{lc}
\multicolumn{1}{c}{ Especies } & Producción de materia seca (t/ha) \\
\hline Centrosema macrocarpum & $9.61^{\mathrm{a}}$ \\
Calopogonium mucunoides & $5.93^{\mathrm{b}}$ \\
Arachis pintoi & $5.07^{\mathrm{b}}$ \\
Canavalia ensiformis & $4.59^{\mathrm{b}}$ \\
Callisia repens & $4.45^{\mathrm{b}}$ \\
\hline Nota: Letras distintas indican diferencias significativas según \\
prueba de Duncan $(\mathrm{p} \leq 0.05)$.
\end{tabular}

\section{c) Biomasa radicular}

En la Tabla 3 se puede observar que las biomasas radiculares de las coberturas fueron significativamente diferentes. La biomasa radicular de Arachis pintoi (3.17 $\left.\mathrm{t} \mathrm{ha}^{-1}\right)$ y Centrosema macrocarpum (2.76 $\left.\mathrm{t} \mathrm{ha}^{-1}\right)$ fueron superiores a las producidas por las otras coberturas. La biomasa radicular es una parte importante de la biomasa de la planta que crece dentro del perfil del suelo, absorbe los nutrientes y el agua para ser transportados a la parte aérea y proporciona el soporte físico para la planta (Sainju et al., 2005). Investigaciones referidas al tema señalan que existe gran variabilidad en la producción de raíces finas y gruesas, según sea el tipo de clima donde se desarrollan, tipo de especie, estado de desarrollo o edad de las plantas. También, factores del suelo son determinantes para explicar las diferencias en productividad, especialmente aquellos que afectan la elongación de las raíces, el abastecimiento de agua y la aireación del suelo. Factores como textura y estructura condicionan las características de la porosidad y drenaje interno, las cuales son importantes al momento de evaluar la fertilidad del suelo para la producción de biomasa subterránea (Guerra et al., 2005).
Tabla 1. Cobertura (\%) de cinco especies vegetales, evaluadas después de la siembra.

\begin{tabular}{|c|c|c|c|c|c|c|c|c|}
\hline \multirow{2}{*}{ Especies } & \multicolumn{7}{|c|}{ Porcentaje de cobertura (DDS*) } & \multirow{2}{*}{$\begin{array}{l}\text { Tasa de } \\
\text { crecimiento } \\
(\% / \text { día })\end{array}$} \\
\hline & 30 & 60 & 90 & 120 & 150 & 180 & 210 & \\
\hline Arachis pintoi & $7.13^{\mathrm{c}}$ & $14.5^{\mathrm{c}}$ & $22.1^{\mathrm{e}}$ & $29.2^{\mathrm{d}}$ & $69.2^{\mathrm{c}}$ & $82.6^{\mathrm{b}}$ & $98.9^{\mathrm{b}}$ & 0.49 \\
\hline Calopogonium mucunoides & $22.0^{\mathrm{b}}$ & $45.5^{\mathrm{b}}$ & $84.3^{\mathrm{b}}$ & $100^{\mathrm{a}}$ & $100^{\mathrm{a}}$ & $100^{\mathrm{a}}$ & $100^{\mathrm{a}}$ & 0.87 \\
\hline Callisia repens & $11.8^{\mathrm{c}}$ & $24.8^{\mathrm{c}}$ & $40.5^{\mathrm{d}}$ & $53.5^{\mathrm{c}}$ & $77.3^{\mathrm{b}}$ & $100^{\mathrm{a}}$ & $100^{\mathrm{a}}$ & 0.55 \\
\hline Canavalia ensiformis & $44.8^{\mathrm{a}}$ & $94.0^{\mathrm{a}}$ & $100^{\mathrm{a}}$ & $100^{\mathrm{a}}$ & $100^{\mathrm{a}}$ & $100^{\mathrm{a}}$ & $100^{\mathrm{a}}$ & 1.16 \\
\hline Centrosema macrocarpum & $9.03^{\mathrm{c}}$ & $19.0^{\mathrm{c}}$ & $63.4^{\mathrm{c}}$ & $83.8^{\mathrm{b}}$ & $100^{\mathrm{a}}$ & $100^{\mathrm{a}}$ & $100^{\mathrm{a}}$ & 0.73 \\
\hline \multicolumn{9}{|c|}{$\begin{array}{l}\text { *Días después de la siembra. } \\
\text { Nota: Letras distintas en las columnas indican diferencias significativas según prueba de Duncan }(\mathrm{p} \leq \\
0.05) \text {. }\end{array}$} \\
\hline \multicolumn{9}{|l|}{ b) Biomasa foliar } \\
\hline
\end{tabular}


aporte de azufre es mayor con C. macrocarpum que contribuye con $14.41 \mathrm{~kg} \mathrm{ha}^{-1}$, superando estadísticamente a los demás cultivos en estudio.

En la Tabla 6, se muestran los aportes potenciales de micronutrientes de cada cobertura en estudio. El mayor aporte de zinc es a través de $A$. pintoi, que alcanza $0.25 \mathrm{~kg} \mathrm{ha}^{-1}$, lo mismo que C. macrocarpum con $0.23 \mathrm{~kg} \mathrm{ha}^{-1}$, mientras que el menor aporte esta dado por C. repens con $0.09 \mathrm{~kg} \mathrm{ha}^{-1}$. Los aportes de cobre fueron menores en comparación a los demás micronutrientes; los valores fluctúan entre 0.02 a 0.12 $\mathrm{kg} \mathrm{ha}{ }^{-1}$, correspondiendo a C. macrocarpum el máximo valor y a $C$. repens el mínimo aporte. $\mathrm{La}$ concentración de manganeso por parte de C. repens, que alcanza $1.34 \mathrm{~kg} \mathrm{ha}^{-1}$, es ampliamente superior a las concentraciones de las demás coberturas en estudio. Los mayores aportes de hierro corresponden a A. pintoi y C. repens, con 2.25 y $1.83 \mathrm{~kg} \mathrm{ha}^{-1}$, mientras que el último microelemento, boro, es aportado en mayores cantidades por $C$. ensiformis y $C$. macrocarpum, difiriendo estadísticamente de las demás coberturas.
Organización de Estados Americanos (CICAD/OEA) y al Departamento de Agricultura de los Estados Unidos de América - Servicio de Investigación Agrícola (USDA-ARS) por el soporte a este proyecto.

\section{Literatura citada}

Alegre J.C., Arévalo A. \& Fasabi R. 2003. Efecto del Fósforo sobre el establecimiento de Centrosema macrocarpum dentro de una plantación de pijuayo Bactris gasipaes en un Ultisol de trópico húmedo. Ecología Aplicada. 2(1): 93-97.

Altieri M. 1999. Agroecología: bases teóricas para una agricultura sustentable. CLADES, Lima, Perú.

Baligar V.C., Elson M.K. \& Meinhardt L.W. 2007. Cover crops useful for improving soil productivity under cacao. USDA-ARS Beltsville Agricultural Research Center, Beltsville. USA.

Canadell J., Jackson R.B., Ehleringer J.R., Mooney H.A. Sala O.E. \& Schulze E.D. 1996. Maximum rooting depth of vegetation types at the global scale. Oecología. 108:583-595.

Espindola J.A., Guerra J.G.M., De-Polli H., De Almeida D.L. \& Abboud A. C. de S. 2005. Adubação verde com leguminosas. Embrapa Informação Tecnológica, Brasilia.

Tabla 6. Extracción total de micronutrientes por cinco especies vegetales García J. \& Duran R. 2000. Evaluación de sistemas usadas como cobertura.

\begin{tabular}{cccccc}
\hline \multirow{2}{*}{ Especies } & \multicolumn{5}{c}{ Micronutrientes $\left(\mathbf{k g ~ h a}^{-1}\right)$} \\
& Zn & Cu & Mn & Fe & B \\
\hline Arachis pintoi & $0.25^{\mathrm{a}}$ & $0.09^{\mathrm{ab}}$ & $0.23^{\mathrm{b}}$ & $2.25^{\mathrm{a}}$ & $0.24^{\mathrm{bc}}$ \\
Calopogonium mucunoides & $0.15^{\mathrm{bc}}$ & $0.08^{\mathrm{b}}$ & $0.17^{\mathrm{b}}$ & $1.45^{\mathrm{b}}$ & $0.27^{\mathrm{b}}$ \\
Callisia repens & $0.09^{\mathrm{c}}$ & $0.02^{\mathrm{c}}$ & $1.34^{\mathrm{a}}$ & $1.83^{\mathrm{ab}}$ & $0.34^{\mathrm{b}}$ \\
Canavalia ensiformis & $0.16^{\mathrm{b}}$ & $0.09^{\mathrm{ab}}$ & $0.13^{\mathrm{b}}$ & $1.61^{\mathrm{b}}$ & $0.67^{\mathrm{a}}$ \\
Centrosema macrocarpum & $0.23^{\mathrm{a}}$ & $0.12^{\mathrm{a}}$ & $0.37^{\mathrm{b}}$ & $1.44^{\mathrm{b}}$ & $0.60^{\mathrm{a}}$
\end{tabular}

de labranza sobre la producción de cultivos en suelos algodoneros del valle del Cesar. Suelos Ecuatoriales. 30(1): 76-85.

Gilbert G. 2002. Interacciones entre microorganismos y plantas. Sección V. En: Guariguata M. \& Catan G. (Eds.). Ecología y conservación de bosques neotropicales. Primera edición. Ediciones LUR. Costa Rica.

Nota: Letras distintas en las columnas indican diferencias significativas según prueba de Duncan $(\mathrm{p} \leq 0.05)$.

\section{Conclusiones:}

1. Con fines de reducir el crecimiento de malezas y proteger al suelo de la erosión, la cobertura Canavalia ensiformis es la más apropiada porque logró el cubrimiento total del suelo a los 90 días después de sembrado.

2. La biomasa total (foliar y radicular) en base a materia seca producida por Centrosema macrocarpum fue superior a la producida por las demás coberturas.

3. Con fines nutricionales y de reciclaje, el mayor aporte potencial de nutrientes se logró con Centrosema macrocarpum ya que presentó la mayor capacidad extractiva de nutrientes del suelo al haber producido más cantidad de materia seca.

\section{Agradecimientos}

A la Sección Antinarcóticos de la Embajada Norteamericana en Perú (NAS - Lima), a la Comisión Interamericana Contra el Abuso de Drogas de la
Guerra C. J., Gayoso A.J., Schlatter V.J. \& Nespolo R.R. 2005. Análisis de la biomasa de raíces en diferentes tipos de bosques: Avances en la evaluación de Pinus radiata en Chile. Bosque (Valdivia). 26(1): 5-21.

Iturregui B.P. 2007. El Perú es muy vulnerable al cambio climático. El Dominical, Entrevista. El Comercio 01 de abril. Lima.

Marinho G.J., Ndiae A., Linhares R. \& Azevedo J.A. 2007. Cultivos de cobertura como indicadores de procesos ecológicos. LEISA. 22(4): 20-22.

Palm C.A, Vostu S.A, Sanchez P.A, Ericksen P.J, (Eds). 2005. Slash and Burn. The Search for Alternatives. Columbia University Press, New York, USA.

Reyes A.C. \& Ara M. 1999. Tasas de siembra y fertilización con $\mathrm{P}$ para el establecimiento de Centrosema macrocarpum en Pucallpa. Revista de Investigaciones Veterinarias del Perú. 10(1).

Reyes A.C., Ara M., Ramos O. \& Clavo Z. 2004. Fertilización con fósforo y control de malezas para el establecimiento de Brachiaria brizantha a escala comercial. Revista de Investigaciones Veterinarias del Perú. 15(2): 92-99.

Sainju U.M., Singh B.P., \& Whitehead W.F. 2005. Tillage, cover crops, and nitrogen fertilization effects on cotton and sorghum root biomass, carbon, and nitrogen. Agronomy Journal. 97:1279-1290. 
${ }^{1}$ Universidad Nacional del Centro del Perú, Facultad de Agronomía, Departamento Académico de Cultivos y Fitomejoramiento, Huancayo, Perú. fernando_puertas@yahoo.com.

${ }^{2}$ Instituto de Cultivos Tropicales (NAS-ICT/CICAD-OEA), Jr. Santa María No 241, Banda de Shilcayo, Tarapoto, Perú. e.arevalo.ict@terra.com.pe, luiszuniga@terra.com.pe

${ }^{3}$ Universidad Nacional Agraria La Molina, Facultad de Agronomía, Departamento Académico de Suelos y Departamento Académico de Fitotecnia, Lima, Perú. jalegre@lamolina.edu.pe, ololi@lamolina.edu.pe, husovi@lamolina.edu.pe

${ }^{4}$ U.S. Department of Agriculture / Agricultural Research Service, Beltsville Agricultural Research Center, Beltsville, MD 20705. VC.Baligar@ars.usda.gov 